\title{
Risk-sensitive probability for Markov chains
}

\author{
Vahid Reza Ramezani, Steven I. Marcus* \\ Department of Electrical and Computer Engineering and Institute for System Research, University of Maryland, \\ 2415 A.V. Williams Building, College Park, MD 20742, USA
}

Received 16 October 2002; received in revised form 1 May 2003; accepted 8 June 2004

Available online 11 November 2004

\begin{abstract}
The probability distribution of a Markov chain is viewed as the information state of an additive optimization problem. This optimization problem is then generalized to a product form whose information state gives rise to a generalized notion of probability distribution for Markov chains. The evolution and the asymptotic behavior of this generalized or "risk-sensitive" probability distribution is studied in this paper and a conjecture is proposed regarding the asymptotic periodicity of risksensitive probability and proved in the two-dimensional case. The relation between a set of simultaneous non-linear and the set of periodic attractors is analyzed.
\end{abstract}

(C) 2004 Elsevier B.V. All rights reserved.

Keywords: Markov chains; Risk-sensitive estimation; Asymptotic periodicity

\section{Introduction}

It is well known that the probability distribution of an ergodic Markov chain is asymptotically stationary, independent of the initial probability distribution, and that the stationary distribution is the solution to a fixedpoint problem (Shiryayev, 1984). This probability

\footnotetext{
Supported in part by the National Science Foundation under Grants DMI-9988867, by the Air Force Office of Scientific Research under Grant F496200110161, and by ONR Contract 01-5-28834 under the MURI Center for Auditory and Acoustics Research.

* Corresponding author. Tel.: 301405 3683; fax: 3014053751.

E-mail addresses: rvahid@isr.umd.edu (V.R. Ramezani), marcus@isr.umd.edu (S.I. Marcus).
}

distribution can be viewed as the information state for an estimation problem arising from the maximum A posterior probability estimator (MAP) estimation of the Markov chain for which no observation is available.

Risk-sensitive filters [1-5,12,13] take into account the "higher-order" moments of the estimation error. Roughly speaking, this follows from the analytic property of the exponential $\mathrm{e}^{x}=\sum_{k=0}^{\infty} x^{k} / k$ ! so that if $\Psi$ stands for the sum of the error functions over some interval of time then

$$
E[\exp (\gamma \Psi)]=E\left[1+\gamma \Psi+(\gamma)^{2}(\Psi)^{2} / 2+\cdots\right] .
$$

Thus, at the expense of the mean error cost, the higherorder moments are included in the minimization of 
the expected cost, reducing the "risk" of large deviations and increasing our "confidence" in the estimator. The parameter $\gamma>0$ controls the extent to which the higher-order moments are included. In particular, the first-order approximation, $\gamma \rightarrow 0, E[\exp (\gamma \Psi)] \cong 1+$ $\gamma E \Psi$, indicates that the original minimization of the sum criterion or the risk-neutral problem is recovered as the small risk limit of the exponential criterion.

Another point of view is that the exponential function has the unique algebraic property of converting the sum into a product. In this paper, we show that a notion of probability for Markov chains follows from this point of view which due to its connection to risk-sensitive filters, will be termed "risk-sensitive probability (RS-probability)". We consider an estimation problem of the states of a Markov chain in which the cost has a product structure. We assume no observation is available and that the initial probability distribution is known. We will define the RS-probability of a Markov chain as an information state for this estimation problem whose evolution is governed by a non-linear operator. The asymptotic behavior of RS-probability appears to be periodic. Asymptotic periodicity has been reported to emerge from random perturbations of dynamical systems governed by constrictive Markov integral operators [6,7]. In our case, the Markov operator is given by a matrix; the perturbation has a simple non-linear structure and the attractors can be explicitly calculated.

In Section 2, we view the probability distribution of a Markov chain as the information state of an additive optimization problem. RS-probability for Markov chains are introduced in Section 3. We show that its evolution is governed by an operator (denoted by $F^{\gamma}$ ) which can be viewed as a generalization of the usual linear Markov operator. The asymptotic behavior of this operator is studied in Section 3 and a conjecture is proposed. Under mild conditions, it appears that RS-probability is asymptotically periodic. This periodic behavior is governed by a set of simultaneous quadratic equations.

\section{Probability as an information state}

In $[9,10]$ we studied the exponential (risk-sensitive) criterion for the estimation of hidden Markov models (HMMs) and introduced risk-sensitive filter banks.

The probability distribution of a Markov chain, knowing only initial distribution, determines the most "likely state" in the sense of MAP. In the context of HMM, the problem can be viewed as that of "pure prediction", i.e., an HMM whose states are entirely hidden.

Define a $\mathrm{HMM}$ as a five-tuple $\langle\mathbf{X}, \mathbf{Y}, \mathbf{X}, \mathscr{A}, Q\rangle$; here $\mathscr{A}$ is the transition matrix, $\mathbf{Y}=\left\{1,2, \ldots, N_{\mathbf{Y}}\right\}$ is the set of observations and $\mathbf{X}=\left\{1,2, \ldots, N_{\mathbf{X}}\right\}$ is the finite set of (internal) states as well as the set of estimates or decisions. In addition, we have that $Q:=$ $\left[q_{x, y}\right]$ is the $N_{\mathbf{X}} \times N_{\mathbf{Y}}$ state/observation matrix, i.e., $q_{x, y}$ is the probability of observing $y$ when the state is $x$. We consider the following information pattern. At decision epoch $t$, the system is in the (unobservable) state $X_{t}=i$ and the corresponding observation $Y_{t}$ is gathered, such that

$$
P\left(Y_{t}=j \mid X_{t}=i\right)=q_{i, j} .
$$

The estimators $V_{t}$ are functions of observations $\left(Y_{0}, \ldots, Y_{t}\right)$ and are chosen according to some specified criterion. Consider a sequence of finite dimensional random variables $X_{t}$ and the corresponding observations $Y_{t}$ defined on the common probability space $(\boldsymbol{\Omega}, M, \mathbf{P})$. Let $\hat{X}_{t}$ be a Borel measurable function of the filtration generated by observations up to $Y_{t}$ denoted by $\mathscr{Y}_{t}$. The MAP is defined recursively; given $\hat{X}_{0}, \ldots, \hat{X}_{t-1}, \hat{X}_{t}$ is chosen such that the following sum is minimized:

$E\left[\sum_{i=0}^{t} \rho\left(X_{i}, \hat{X}_{i}\right)\right]$,

where

$\rho(u, v)= \begin{cases}0 & \text { if } u=v, \\ 1 & \text { otherwise. }\end{cases}$

The usual definition of MAP as the argument with the greatest probability given the observation follows from the above [8]. The solution is well known; we need to define recursively an information state

$\sigma_{t+1}=N_{\mathbf{Y}} \cdot \bar{Q}\left(Y_{t+1}\right) \mathscr{A}^{\mathrm{T}} \cdot \sigma_{t}$,

where $\bar{Q}(y):=\operatorname{diag}\left(q_{i, y}\right), \mathscr{A}^{\mathrm{T}}$ denotes the transpose of the matrix $\mathscr{A}$. $\sigma_{0}$ is set equal to $N_{\mathbf{Y}} \cdot \bar{Q}\left(Y_{0}\right) p_{0}$, 
where $p_{0}$ is the initial distribution of the state and is assumed to be known. Note that (3) is not normalized.

When no observation is available, it is easy to see that $N_{\mathbf{Y}} \cdot \bar{Q}\left(Y_{t}\right)=I$, where $I$ is the identity matrix. Thus, the information state for the prediction case evolves according to $\sigma_{t+1}=\mathscr{A}^{\mathrm{T}} \cdot \sigma_{t}$ which when normalized is simply the probability distribution of the chain.

This "prediction" optimization problem for a multiplicative cost will be considered next.

\section{RS-probability for Markov chains}

With the notation of the previous section, given $\hat{X}_{0}, \ldots, \hat{X}_{t-1}$, define $\hat{X}_{t}$ recursively as the estimator which minimizes the exponential (risk-sensitive) cost

$E\left[\exp \left\{\gamma \sum_{i=0}^{t} \rho\left(X_{i}, \hat{X}_{i}\right)\right\}\right]$,

where $\gamma$ is a strictly positive (risk-sensitive) parameter.

As discussed in the introduction, the exponential criterion allows for the inclusion of higherorder moments of the cost and the approximation $E[\exp (\gamma \Psi)] \cong 1+\gamma E \Psi$ shows that for small values of $\gamma$, the additive cost criterion is recovered. The structure of $\rho$ allows for the following simplification of (4):

$E\left[\prod_{i=0}^{t} \rho^{*}\left(X_{i}, \hat{X}_{i}\right)\right]$,

$\rho^{*}(u, v)= \begin{cases}1 & \text { if } u=v, \\ r=\mathrm{e}^{\gamma} & \text { otherwise. }\end{cases}$

Define an information state

$\sigma_{t+1}^{\gamma}=N_{\mathbf{Y}} \cdot \bar{Q}\left(Y_{t+1}\right) \mathscr{D}^{\mathrm{T}}\left(\hat{X}_{t}\right) \cdot \sigma_{t}^{\gamma}$,

where $\bar{Q}(y):=\operatorname{diag}\left(q_{i, y}\right), \mathscr{A}^{\mathrm{T}}$ denotes the transpose of the matrix $\mathscr{A}$ and the matrix $\mathscr{D}$ is defined by

$[\mathscr{D}(v)]_{i, j}:=a_{i, j} \exp (\gamma \rho(i, v))$.

$\sigma_{0}^{\gamma}$ is set equal to $N_{\mathbf{Y}} \cdot \bar{Q}\left(Y_{0}\right) p_{0}$, where $p_{0}$ is the initial distribution of the state and is assumed to be known. The proof of the following theorem can be found in [9].
Theorem 1. The optimization problem (4) is solved recursively by

$\hat{X}_{t}(\sigma)=i \quad$ if $\sigma^{i} \geqslant \sigma^{j}, \quad \forall j \neq i$,

where $\sigma=\left(\sigma^{1}, \ldots, \sigma^{N_{\mathbf{X}}}\right)$ is the value the information state (6) takes at time $t$.

We next obtain a simplex preserving operator $F^{\gamma}$ by assuming that no observation is available and that the initial probability distribution is given. In the riskneutral context, this operator is simply $\mathscr{A}^{\mathrm{T}}$ which governs the evolution of probability distribution; as the risk-sensitive cost is a generalization of the riskneutral one, one might expect that this new operator which governs the evolution of "risk-sensitive probability" to be a generalization of $\mathscr{A}^{\mathrm{T}}$. Setting $N_{\mathbf{Y}} \cdot \bar{Q}\left(Y_{t}\right)$ equal to the identity matrix $I$ corresponds to the case when no observation is available. It can be shown that the information state is independent of scaling, i.e., if $\sigma$ is an information state so is $\alpha \sigma$ for every $\alpha>0$ and replacing it one with the other does not change the resulting estimate of the state. Associate with each $i \in X$, a unit vector in $R_{\mathbf{X}}^{N}$ whose $i$ th component is 1 . Denote the "risk-sensitive probability" $U_{t}$ as the normalized information state (6) when no observation is available. The proof of the following theorem can be found in [9].

Theorem 2. Let $N_{\mathbf{Y}} \cdot \bar{Q}\left(Y_{t}\right)=I$, then the estimator which minimizes (5) is given by

$\hat{X}_{t}=\arg \max _{\left[i \in \mathbf{S}_{\mathbf{X}}\right]}\left\langle U_{t}, e_{i}\right\rangle$,

where $U_{t}$ evolves according to

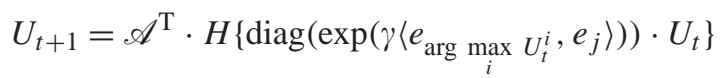

$$
\begin{aligned}
& :=F^{\gamma}\left(U_{t}\right),
\end{aligned}
$$

and $H(X)=X / \sum_{i}\left(X_{i}\right)$ and $U_{0}=p_{0}$.

The operator $F^{\gamma}$ can be viewed as a non-linear generalization of the linear operator $\mathscr{A}^{\mathrm{T}}$. It is apparent that this operator plays the same role in the context of risk-sensitive estimation as the operator $\mathscr{A}^{\mathrm{T}}$ does in the risk-neutral case. Thus, one might expect that the risk-sensitive properties of the exponential criterion be reflected in the action of $F^{\gamma}$.

First, observe that both operators are simplex preserving and $F^{\gamma} \rightarrow \mathscr{A}^{\mathrm{T}}$ as $\gamma \rightarrow 0$. It is well known 
that under primitivity of the matrix $\mathscr{A}$, the dynamical system defined by

$p_{n+1}=\mathscr{A}^{\mathrm{T}} p_{n}$,

for every choice of the initial probability distribution $p_{0}$, converges to $p^{*}$ which satisfies $\mathscr{A}^{\mathrm{T}} p^{*}=p^{*}[11]$.

Definition. A cycle of RS-probability (CRP) is a finite set of probabilities $\left\{v^{1}, \ldots, v^{m}\right\}$ such that $F^{\gamma}\left(v^{i}\right)=$ $v^{i+1}$ with $F^{\gamma}\left(v^{m}\right)=v^{1} ; m$ is called the period of the CRP.

We pose the following conjecture:

Conjecture. Let the stochastic matrix $\mathscr{A}$ be primitive. Then, for every choice of the initial probability distribution $p_{0}$, the dynamical system

$U_{t+1}=F^{\gamma}\left(U_{t}\right)$

is asymptotically periodic, i.e., $U_{t}$ approaches a CRP as $t \rightarrow \infty$ satisfying the equations

$F^{\gamma}\left(v^{1}\right)=v^{2}, F^{\gamma}\left(v^{2}\right)=v^{3}, \ldots, F^{\gamma}\left(v^{m}\right)=v^{1}$.

The condition $F^{\gamma}\left(v^{1}\right)=v^{2}, F^{\gamma}\left(v^{2}\right)=v^{3}, \ldots, F^{\gamma}\left(v^{m}\right)$ $=v^{1}$ can be considered a generalization of the equation $\mathscr{A}^{\mathrm{T}} p^{*}=p^{*}$. It is not difficult to show that in general, the equations are quadratic. Note that we do not exclude the case $m=1$; the CRP only has one element and thus $F^{\gamma}$ is asymptotically stationary.

In the appendix, we give sufficient conditions under which the conjecture holds in two dimensions with a CRP which is independent of the initial point.

Next, we report a number of other properties of $F^{\gamma}$.

Property 1 (Dependence of the asymptotic behavior on the initial condition). The asymptotic behavior of $F^{\gamma}$ may depend on the initial conditions. That is, depending on the initial condition a different CRP may emerge. Let $\mathscr{A}$ be given by

$\mathscr{A}=\left[\begin{array}{ll}0.2 & 0.8 \\ 0.6 & 0.4\end{array}\right], \quad \mathrm{e}^{\gamma}=100$.

Let the initial condition be given by $\left(u_{1}, u_{2}\right)$. There are two different CRPs depending on the initial conditions

$F^{\gamma}(u)=u=\left[\begin{array}{l}0.594 \\ 0.405\end{array}\right] \quad$ if $u_{1} \geqslant u_{2}$,
$F^{\gamma}(v)=v=\left[\begin{array}{l}0.214 \\ 0.785\end{array}\right] \quad$ if $u_{2}>u_{1}$

When is the asymptotic behavior independent of the initial condition? We believe this depends on the relation between the diagonal and off-diagonal elements of $\mathscr{A}$. For example, consider the matrix

$\mathscr{A}=\left[\begin{array}{ll}0.6 & 0.4 \\ 0.25 & 0.75\end{array}\right], \quad \mathrm{e}^{\gamma}=10$.

The CRP, for every initial condition, has two elements

$C R P:\left(v^{1}, v^{2}\right), F^{\gamma}\left(v^{1}\right)=v^{2}, F^{\gamma}\left(v^{2}\right)=v^{1}$.

$v^{1}=\left[\begin{array}{l}0.283 \\ 0.716\end{array}\right], \quad v^{2}=\left[\begin{array}{l}0.534 \\ 0.465\end{array}\right]$.

It appears that when the diagonal elements "dominate" the off-diagonal elements, the asymptotic behavior is independent of the initial condition. We have carried out a thorough investigation for $6 \times 6$ stochastic matrices and lower dimensions, but we suspect the property holds in higher dimensions. But, below we describe some special cases.

Property 2 (Dependence of the period on $\gamma$ ). Our simulations show that for small values of $\gamma$ the period is 1, i.e., $F^{\gamma}$ is asymptotically stationary. As $\gamma$ increases periodic behavior may emerge; based on simulation of the examples we have studied, the period tends to increase with increasing $\gamma$ but then decrease for large values. So, the most complex behavior occurs for the mid-range values of $\gamma$. Consider

$\mathscr{A}=\left[\begin{array}{ll}0.8 & 0.2 \\ 0.4 & 0.6\end{array}\right]$,

and let $m$ be the period. Our simulations show that the period $m$ of the CRPs depends on the choice of $\gamma$; our simulations results in the pairs $\left(\mathrm{e}^{\gamma}, m\right):(2.1,1)$ $(2.7,1)(2.9,1)(3,1)(3.01,7)(3.1,5)(3.3,4)(3.9,3)$ $(10,2)(21,2)$. We can see that even in two dimensions, the behavior of $F^{\gamma}$ is complex.

When does the periodic behavior emerge? The fixed-point problem provides the answer. If the fixedpoint problem $F^{\gamma}(u)=u$ does not have a solution satisfying $0 \leqslant u \leqslant 1$, the asymptotic behavior cannot be stationary. For two dimensions, the equation 


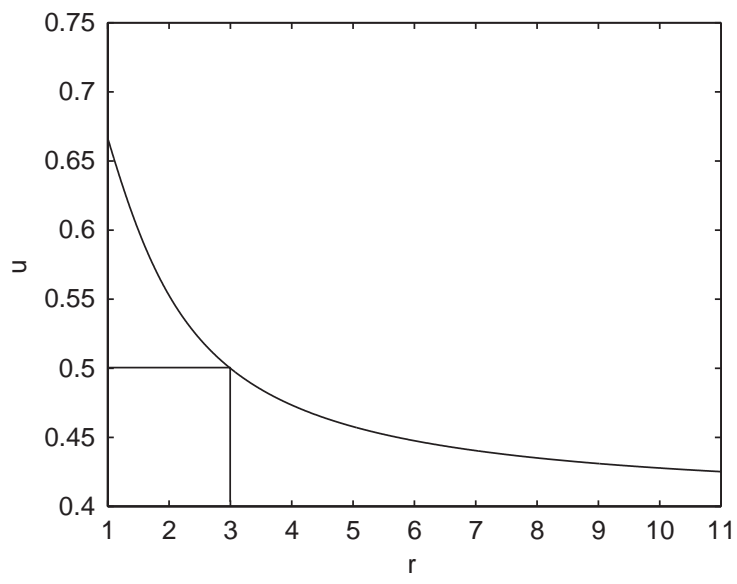

Fig. 1. The emergence of periodicity.

$F^{\gamma}(u)=u=\left(u_{1}, u_{2}\right)^{\mathrm{T}}$ is easy to write. Assume $u_{1}>u_{2}$ (for the case $u_{2}>u_{1}$, we transpose 1 and 2).

$\mathscr{A}=\left[\begin{array}{ll}a_{11} & a_{12} \\ a_{21} & a_{22}\end{array}\right]$,

and recall that $u_{1}+u_{2}=1$. This yields

$$
\begin{aligned}
& \left(\mathrm{e}^{\gamma}-1\right) u_{1}^{2}+u_{1}\left(a_{11}-\mathrm{e}^{\gamma} a_{21}-\mathrm{e}^{\gamma}\right) \\
& +a_{21} \mathrm{e}^{\gamma}=0, \quad u_{1} \geqslant u_{2}, \\
& \left(\mathrm{e}^{\gamma}-1\right) u_{2}^{2}+u_{2}\left(a_{22}-\mathrm{e}^{\gamma} a_{12}-\mathrm{e}^{\gamma}\right) \\
& +a_{12} \mathrm{e}^{\gamma}=0, \quad u_{2}>u_{1} .
\end{aligned}
$$

First, note that when $\gamma=0$, we have

$u_{1}\left(a_{12}+a_{21}\right)=a_{21}$

which is linear and is the fixed-point problem $\mathscr{A}^{\mathrm{T}}(u)=$ $u$. For the above example, the roots of the equation resulting from the assumption $u_{2}>u_{1}$ are greater than one for all ranges of $\mathrm{e}^{\gamma}>1$. Thus, stationarity requires that a solution to

$$
\begin{gathered}
\left(\mathrm{e}^{\gamma}-1\right) u_{1}^{2}+u_{1}\left(0.8-\mathrm{e}^{\gamma} 0.4-\mathrm{e}^{\gamma}\right) \\
+0.4 \mathrm{e}^{\gamma}=0, \quad u_{2}<u_{1},
\end{gathered}
$$

exist. One solution turns out to be greater than 1 . The other solution is plotted vs. $r=\mathrm{e}^{\gamma}$ in Fig. 1. The condition $u_{2}<u_{1}$ fails for $\mathrm{e}^{\gamma}>3$. Thus for $\mathrm{e}^{\gamma}>3$ no stationary solution can exist. If the conjecture is correct, the periodic behavior must emerge, which is exactly what we observed above. Based on the examples we have studied, this is a general property of $F^{\gamma}$ in two dimensions when diagonal elements "dominate".

Let $a_{11}>a_{12}$ and $a_{22}>a_{21}$. Also, assume without loss of generality, that $a_{11}>a_{22}$. For the stationary solution to exist as we showed above, (20) must have a solution. Let $\Delta=a_{11}-\mathrm{e}^{\gamma} a_{21}-\mathrm{e}^{\gamma}$. For small values of $\gamma$, the probability solution of $(20)\left(0 \leqslant u_{1} \leqslant 1\right)$ turns out to be

$\frac{-\Delta-\sqrt{\Delta^{2}-4 a_{21} \mathrm{e}^{\gamma}\left(\mathrm{e}^{\gamma}-1\right)}}{2\left(\mathrm{e}^{\gamma}-1\right)}$,

and as $u_{2}<u_{1}$ implies $\frac{1}{2}<u_{1}$, we must have

$\frac{-\Delta-\sqrt{\Delta^{2}-4 a_{21} \mathrm{e}^{\gamma}\left(\mathrm{e}^{\gamma}-1\right)}}{2\left(\mathrm{e}^{\gamma}-1\right)}>\frac{1}{2}$,

which after some simple algebra implies

$\mathrm{e}^{\gamma}<\frac{2 a_{11}-1}{1-2 a_{21}}$.

If we plug in $a_{11}=0.8$ and $a_{21}=0.4$, we get $\mathrm{e}^{\gamma}<3$. If the conjecture is true, periods must appear for $\mathrm{e}^{\gamma}>3$. At $\mathrm{e}^{\gamma}=\left(2 a_{11}-1\right) /\left(1-2 a_{21}\right)$, we get $u_{1}=u_{2}=\frac{1}{2}$ which can be shown to be an acceptable stationary solution; hence $\left(2 a_{11}-1\right) /\left(1-2 a_{21}\right)$ is a sharp threshold. Our computations have been consistent with this result. For the case $a_{11}<a_{22}$, we obtain

$\mathrm{e}^{\gamma}<\frac{2 a_{22}-1}{1-2 a_{12}}$.

Writing $a_{i i}=\frac{1}{2}+\varepsilon$ and $a_{j i}=\frac{1}{2}-\delta$, both results can be written as

$\mathrm{e}^{\gamma}<\frac{\varepsilon}{\delta}$

Eq. (27) is a measure of sensitivity to risk.

Periodicity seems persistent; once the periodic solutions emerge, increasing $\mathrm{e}^{\gamma}$ does not seem to bring back the stationary behavior. In two dimensions for large values of $\mathrm{e}^{\gamma}$, an interesting classification is possible. Given that the conjecture holds, an obvious sufficient condition for periodicity would be for the roots of (20) and (21) to be complex:

$$
\begin{aligned}
& \left(a_{11}-\mathrm{e}^{\gamma} a_{21}-\mathrm{e}^{\gamma}\right)^{2}-4\left(\mathrm{e}^{\gamma}-1\right) a_{21} \mathrm{e}^{\gamma}<0, \\
& \left(a_{22}-\mathrm{e}^{\gamma} a_{12}-\mathrm{e}^{\gamma}\right)^{2}-4\left(\mathrm{e}^{\gamma}-1\right) a_{12} \mathrm{e}^{\gamma}<0 .
\end{aligned}
$$


But, further inspection shows for sufficiently large values of $e^{\gamma}$, the inequalities give

$\mathrm{e}^{2 \gamma}\left(1-a_{21}\right)^{2}<0$

$\mathrm{e}^{2 \gamma}\left(1-a_{12}\right)^{2}<0$

which are clearly false and so real roots exist. Other relations can be exploited to show that these roots are unacceptable and hence demonstrate the existence of periodic attractors as we will show next. Consider the case where $\mathrm{e}^{\gamma} \gg a_{i j}, 0<a_{i j}<1$. Then, the fixed-point problem (20) can be written as

$\mathrm{e}^{\gamma} u_{1}^{2}-\mathrm{e}^{\gamma}\left(1+a_{21}\right) u_{1}+a_{21} \mathrm{e}^{\gamma}=0$,

$u_{1}^{2}-u_{1}\left(1+a_{21}\right)+a_{21}=\left(u_{1}-1\right)\left(u_{1}-a_{21}\right)$.

The solutions turn out to be $(1,0)$ and $\left(a_{21}, a_{22}\right)$. $(1,0)$ can be ruled out by the assumption $0<a_{i j}<1$. The assumption $u_{1}<u_{2},(21)$, leads by transposition to solutions $(1,0)$ and $\left(a_{11}, a_{12}\right)$. Thus, if we assume $a_{11}>a_{12}$ and $a_{22}>a_{21}$, both solutions can be ruled out; for large values of $\mathrm{e}^{\gamma}$ the fixed-point value problem with period one (the stationary case) does not have a solution and the period must be two or more.

If we assume $a_{21}>a_{22}$ and $a_{12}>a_{11}$ then both $\left(a_{21}, a_{22}\right)$ and $\left(a_{11}, a_{12}\right)$ are acceptable solutions. This was the case in (13) and (14); our computations show that there are in fact two stationary solutions close to $\left(a_{21}, a_{22}\right)$ and $\left(a_{12}, a_{11}\right)$ depending on the initial conditions. Likewise, we can use the simultaneous quadratic equations to classify all the attractors in two dimensions which emerge with increasing $\mathrm{e}^{\gamma}$. For the

\begin{tabular}{|l|c|c|}
\cline { 2 - 3 } \multicolumn{1}{c|}{} & period & attractors \\
\hline$a_{i i}<a_{i j} \quad i \neq j$ & 1 & 2 \\
\hline$a_{i i}>a_{i j} \quad i \neq j$ & 2 & 2 \\
\hline$a_{i i}<a_{i j \quad a_{i \neq j}>a_{j i}}$ & 1 & 1 \\
\hline
\end{tabular}

Fig. 2. The classification in two dimensions.

matrix $\mathscr{A}$ given by (18), we see that this behavior is already emergent at about $\mathrm{e}^{\gamma}=10$. Fig. 2 shows the classification. It is possible to write down these equations in higher dimensions as simultaneous quadratic equations parameterized by $\mathrm{e}^{\gamma}$. Classifying the solutions of these equations is an interesting open problem.

\section{Conclusions}

The risk-sensitive estimation of HMMs gives rise to a notion of probability for Markov chains arising from a non-linear generalization of the linear operator $\mathscr{A}^{\mathrm{T}}$, where $\mathscr{A}$ is a row-stochastic primitive matrix. This operator, denoted by $F^{\gamma}$ in this paper, has a number of properties summarized in the table above. There is an interesting relation between the asymptotic behavior of $F^{\gamma}$ and a set of simultaneous non-linear equations parameterized by $\mathrm{e}^{\gamma}$ determining the periodic solutions (Fig. 3). We have studied the two-dimensional case in this paper and provided analytic and numerical results.

\begin{tabular}{|l|c|c|c|c|}
\hline & Cost & evolution & Asym. & attractors \\
\hline Probability & additive & linear & $\begin{array}{l}\text { asym. } \\
\text { stationarity }\end{array}$ & $\begin{array}{c}\mathrm{A}^{\mathrm{T}} \mathrm{p}=\mathrm{p} \\
\text { Independent of initial conditions }\end{array}$ \\
\hline RS-Probability & multiplicative & non-linear & $\begin{array}{l}\text { asym. } \\
\text { periodicity }\end{array}$ & $\begin{array}{l}\mathrm{F}^{\gamma} \mathrm{v} 1=\mathrm{v} 2, \mathrm{~F}^{\gamma} \mathrm{v} 2=\mathrm{v} 3 \ldots . \mathrm{F}^{\gamma} \mathrm{vm}=\mathrm{v} 1 \\
\text { Dependent on initial conditions }\end{array}$ \\
\hline
\end{tabular}

Fig. 3. Comparing $F^{\gamma}$ and $\mathscr{A}^{\mathrm{T}}$. 
We have posed a series of open problems which are the subject of our further research.

\section{Acknowledgements}

The authors would like to thank an anonymous reviewer for significant comments and suggestions.

\section{Appendix}

We say a function $f(x)$ defined on $D \subset R^{n}$ is asymptotically periodic at $p_{0}$ with period $r$ if there is a set $R\left(p_{0}\right)=\left\{x_{0}, \ldots, x_{r-1}\right\}, x_{i} \neq x_{j}$ if $i \neq j$ and such that, for any $\varepsilon>0$,

$\left|f^{m}\left(p_{0}\right)-x_{[m]}\right|<\varepsilon \quad$ for $m \geqslant N(\varepsilon)$,

where $[m]$ is the remainder of division $m / r, x_{[m]} \in$ $R\left(p_{0}\right)$ and when $R\left(p_{0}\right)$ has only one element, we say that the function is asymptotically stationary. If all points in $R\left(p_{0}\right)$ belong to the simplex we refer to $R\left(p_{0}\right)$, as a cycle of RS-probability or a CRP.

We say that a function $f(x)$ defined on $D$ is asymptotically periodic if it is asymptotically periodic at every point of $D$. Finally a uniquely asymptotically periodic function on $D$ is an asymptotically periodic function such that $R(x)$ for $\forall x \in D$ is unique up to a permutation.

Recall that RS-probability is the normalized information state and thus in the two-dimensional case belongs to the 1-simplex while the unnormalized information state takes its values in the first quadrant of $R^{2}$. Also recall that the information state has the scale independence property, i.e, all points on a radial line through the origin (excluding the origin) produce the same estimate of the state when considered as the value of the information state. Thus, we can go back and forth between the first quadrant of $R^{2}$ and the 1simplex without difficulty. In the proof of the theorem certain linear operators acting on the information state are extended to all of $R^{2}$ for mathematical simplicity. Throughout the proof, a "cone" is the area that lies between two radial lines through the origin always restricted to the first quadrant and unless stated otherwise, it includes the two radial lines. The cone defined by $x=0$ and $y$ will be denoted by LC and the cone defined by the $Y$-axis and $x=y$ by UC.
$F^{\gamma}$ in (8) is non-linear and is not continuous on the simplex but fortunately it is piecewise continuous and in two dimensions it can be written as

$F^{\gamma}(W)= \begin{cases}\mathscr{A}^{\mathrm{T}} H\left(\left[\begin{array}{ll}1 & 0 \\ 0 & \mathrm{e}^{\gamma}\end{array}\right]\right) W, & W^{1} \geqslant W^{2}, \\ \mathscr{A}^{\mathrm{T}} H\left(\left[\begin{array}{cc}\mathrm{e}^{\gamma} & 0 \\ 0 & 1\end{array}\right]\right) W, & \text { otherwise. }\end{cases}$

We will define

$E_{1}:=\mathscr{A}^{\mathrm{T}}\left[\begin{array}{ll}1 & 0 \\ 0 & \mathrm{e}^{\gamma}\end{array}\right], \quad E_{2}:=\mathscr{A}^{\mathrm{T}}\left[\begin{array}{ll}\mathrm{e}^{\gamma} & 0 \\ 0 & 1\end{array}\right]$

and $E:=E_{2} E_{1}$.

The properties of $E$ will be of importance as we shall see.

Note that $\mathscr{A}^{\mathrm{T}}$ is stochastic and thus $\mathscr{A}^{\mathrm{T}} H(X)=$ $H\left(\mathscr{A}^{\mathrm{T}} X\right)$. We will use this property throughout.

Theorem A.1. Suppose the transition probability matrix $\mathscr{A}$ is given by

$\mathscr{A}^{\mathrm{T}}=\left[\begin{array}{ll}1-\varepsilon & \delta \\ \varepsilon & 1-\delta\end{array}\right]$

with $0<\varepsilon<1 / 2$ and $0<\delta<\frac{1}{2}$. Then, for sufficiently large $\gamma, F^{\gamma}$ is uniquely asymptotically periodic with period 2 .

Proof. The proof will be given in two parts. We first show that for sufficiently large values of $\gamma$, there is an invariant "oscillating cone "(OC) in which the information state under the action of $F^{\gamma}$ alternates between $\mathrm{OC} 1=\mathrm{OC} \cap \mathrm{LC}$ and $\mathrm{OC} 2=\mathrm{OC} \cap \mathrm{UC}$, i.e., if $F^{\gamma}(x)=y$ and $x$ belongs to $\mathrm{OC} 1$, then $y$ is in $\mathrm{OC} 2$ and vise versa. We then prove that starting from any point in OC, $F^{\gamma}$ restricted to $\mathrm{OC}$ is uniquely asymptotically periodic.

In the second part, we show that if the information state starts from any point outside OC, we will end up in OC.

In order to carry out the first part of the proof, we need to understand the asymptotic behavior of the 
following linear operator. Its significance will become clear shortly.

$$
\begin{aligned}
E= & {\left[\begin{array}{ll}
1-\varepsilon & \delta \\
\varepsilon & 1-\delta
\end{array}\right]\left[\begin{array}{ll}
\mathrm{e}^{\gamma} & 0 \\
0 & 1
\end{array}\right] } \\
& \times\left[\begin{array}{ll}
1-\varepsilon & \delta \\
\varepsilon & 1-\delta
\end{array}\right]\left[\begin{array}{ll}
1 & 0 \\
0 & \mathrm{e}^{\gamma}
\end{array}\right] .
\end{aligned}
$$

We will show that for sufficiently large values of $\gamma, \mathrm{E}$ has two real positive unequal eigenvalues, say $\lambda_{1}>\lambda_{2}$. The corresponding eigenvectors $V_{1}$ and $V_{2}$ lie in the first and the fourth quadrant, respectively.

The characteristic polynomial of $E$ is given by

$$
\begin{aligned}
p(\lambda)= & \lambda^{2}-\left[\varepsilon \delta \mathrm{e}^{2 \gamma}+\mathrm{e}^{\gamma}(1-\varepsilon)^{2}+\mathrm{e}^{\gamma}(1-\delta)^{2}\right. \\
& +\varepsilon \delta] \lambda+\mathrm{e}^{2 \gamma}(1-\varepsilon-\delta)^{2} .
\end{aligned}
$$

We denote $b=\left[\varepsilon \delta \mathrm{e}^{2 \gamma}+\mathrm{e}^{\gamma}(1-\varepsilon)^{2}+\mathrm{e}^{\gamma}(1-\delta)^{2}+\varepsilon \delta\right]$ and $c=\mathrm{e}^{2 \gamma}(1-\varepsilon-\delta)^{2}$.

The eigenvalues are given by $\lambda_{i}=\frac{1}{2}\left(b \pm\left(b^{2}-\right.\right.$ $4 c)^{1 / 2}$ ), where $\lambda_{1}$ is the root with the positive sign in place of \pm and $\lambda_{2}$ is the root with the negative sign. As $\mathscr{A}$ is primitive, we have $\varepsilon \delta>0$ and $b^{2}$ is of order $\mathrm{e}^{4 \gamma}$ while $c$ is of order $\mathrm{e}^{2 \gamma}$. It follows that for large enough $\gamma$, the roots are real. We have $c=\lambda_{1} \lambda_{2}>0$ and $b=\lambda_{1}+\lambda_{2}>0$. Thus both roots are positive.

The first-order square root binomial expansion for $(1+z)^{1 / 2}=(1+z / 2+\mathrm{o}(z))(\mathrm{o}(z)$ indicates secondorder term and higher terms) holds for $|z|<1$. After a few simple steps we can write

$\lambda_{i}=1 / 2\left[b \pm b\left(1-4 c / 2 b^{2}+\mathrm{o}\left(4 c / b^{2}\right)\right)\right]$.

We have, for sufficiently large values of $\gamma$,

$$
\begin{aligned}
\lambda_{1} & =b-c / b+b / 2 \cdot \mathrm{o}\left(4 c / b^{2}\right)>\lambda_{2} \\
& =c / b-b / 2 \cdot \mathrm{o}\left(4 c / b^{2}\right) .
\end{aligned}
$$

We next show that the eigenvector corresponding to $\lambda_{1}$ lies in the first quadrant (or equivalently, the third) while the eigenvector corresponding to $\lambda_{2}$ lies in the fourth (or equivalently, the second).

Observe that $E$ preserves the first quadrant. Moreover, it is apparent from (35) that $E$ is the product of two diagonal matrices and a stochastic primitive matrix appearing twice. Multiplication by $\mathscr{A}^{\mathrm{T}}$ guarantees that the resulting vector has positive components. As $\mathrm{e}^{\gamma}>1$, the diagonal matrices increase the sum of the components, one by multiplying the first component by $\mathrm{e}^{\gamma}$ and the other by multiplying the second component by $\mathrm{e}^{\gamma}$ while $\mathscr{A}^{\mathrm{T}}$, being stochastic, preserves the sum of the components. Hence, the sum of the components of all vectors in the first quadrant can be made arbitrarily large under the action of $E$ by choosing $\gamma$ large. This means $V_{2}$, the eigenvector corresponding to $\lambda_{2}$, cannot lie in the first quadrant because of the following contradiction:

Suppose $V_{2}$ lies in the first quadrant.

$$
\begin{aligned}
& E V_{2}=\lambda_{2} V_{2}=\frac{c}{\lambda_{1}} V_{2}= \\
& \frac{\mathrm{e}^{2 \gamma}(1-\varepsilon-\delta)^{2}}{\varepsilon \delta \mathrm{e}^{2 \gamma}+\mathrm{e}^{\gamma}(1-\varepsilon)^{2}+\mathrm{e}^{\gamma}(1-\delta)^{2}+\varepsilon \delta-\frac{c}{b}+\frac{b}{2} \mathrm{o}\left(\frac{4 c}{b^{2}}\right)} V_{2}
\end{aligned}
$$

and as $\gamma \rightarrow \infty$,

$\lambda_{2} \rightarrow \frac{(1-\varepsilon-\delta)^{2}}{\varepsilon \delta}$.

On the other hand, the sum of the components of $E V_{2}$ can be shown to be $\left(\mathrm{e}^{\gamma}(1-\varepsilon)+\varepsilon\right) V_{2}^{1}+\left(\delta \mathrm{e}^{2 \gamma}+(1-\right.$ $\left.\delta) \mathrm{e}^{\gamma}\right) V_{2}^{2}$ and so we must have

$$
\begin{aligned}
& {\left[\left(\mathrm{e}^{\gamma}(1-\varepsilon)+\varepsilon\right)-\lambda_{2}\right] V_{2}^{1}} \\
& \quad+\left[\left(\delta \mathrm{e}^{2 \gamma}+(1-\delta) \mathrm{e}^{\gamma}\right)-\lambda_{2}\right] V_{2}^{2}=0 .
\end{aligned}
$$

Clearly if $V_{2}$ belongs to the first quadrant, one of its components must be strictly positive and the other non-negative. But, (36) implies that we can choose $\gamma$ large enough so that $\left[\left(\mathrm{e}^{\gamma}(1-\varepsilon)+\varepsilon\right)-\lambda_{2}\right]>0$ and $\left[\left(\delta \mathrm{e}^{2 \gamma}+(1-\delta) \mathrm{e}^{\gamma}\right)-\lambda_{2}\right]>0$ and thus the above equality cannot hold, a contradiction.

To show that $V_{1}$ must lie in the first quadrant, observe that any vector $W$ can be written as

$W=\alpha_{1} V_{1}+\alpha_{2} V_{2}$,

and

$$
\begin{aligned}
E^{t} W & =\alpha_{1} \lambda_{1}^{t} V_{1}+\alpha_{2} \lambda_{2}^{t} V_{2} \\
& =\lambda_{1}^{t}\left(\alpha_{1} V_{1}+\alpha_{2}\left(\lambda_{2} / \lambda_{1}\right)^{t} V_{2}\right) .
\end{aligned}
$$

As $t \rightarrow \infty,\left(\lambda_{2} / \lambda_{1}\right)^{t} \rightarrow 0$. Now assume $W$ is in the first quadrant. Since we showed that $V_{2}$ does not belong to the first quadrant, $E^{t} W$ will approach a positive multiple of the "dominant eigenvector" $V_{1}$. But $E$ preserves the first quadrant and thus $E^{t} W$ can never exit the first quadrant. Thus, $V_{1}$ must lie in the first quadrant. Later on, we will use (37) to show the existence and the uniqueness of the periodic orbit. 
We are now in position to carry out the first part of the proof. First, we show the existence of the "oscillating cone" OC and using properties of $E$ shown above, prove the unique asymptotic periodicity of $F^{\gamma}$. We consider the lower cone LC first; the situation for $\mathrm{UC}$ is similar.

The normalization function $H(\cdot)$ in $(8)$ restricts the information state to the simplex, but recall that the information state has the scale independence property and thus we can drop the normalization $H(\cdot)$ in (8) and still obtain an information state. To recover the normalized quantity, we can apply $H(\cdot)$ at any desired time step and recover the normalized information state (RS-probability). In what follows, we denote the operator without normalization function $H(\cdot)$ with script notation $\mathscr{F}^{\gamma}$ defined as

$\mathscr{F}^{\gamma}(W)=\left\{\begin{array}{l}\mathscr{A}^{\mathrm{T}}\left[\begin{array}{ll}1 & 0 \\ 0 & \mathrm{e}^{\gamma}\end{array}\right] W, \quad W \in \mathrm{LC}, \\ \mathscr{A}^{\mathrm{T}}\left[\begin{array}{ll}\mathrm{e}^{\gamma} & 0 \\ 0 & 1\end{array}\right] W, \quad W \in \mathrm{UC} .\end{array}\right.$

We would like to show the existence of a cone inside LC which is mapped to UC under the action of $\mathscr{F} \gamma$. On LC, $\mathscr{F}^{\gamma}=E_{1}$. This means that for $W \in \mathrm{LC}$

$E_{1} W=\left[\begin{array}{l}(1-\varepsilon) W_{1}+\delta \mathrm{e}^{\gamma} W_{2} \\ \varepsilon W_{1}+(1-\delta) \mathrm{e}^{\gamma} W_{2}\end{array}\right]$.

If $(1-\varepsilon) W_{1}+\delta \mathrm{e}^{\gamma} W_{2}<\varepsilon W_{1}+(1-\delta) \mathrm{e}^{\gamma} W_{2}$, then $W$ is mapped to UC. This can be written as

$(1-2 \varepsilon) W_{1}<\mathrm{e}^{\gamma}(1-2 \delta) W_{2}$.

Because $\delta<1 / 2$, we can divide both side by $(1-2 \delta)$ without changing the direction of the inequality.

$\frac{(1-2 \varepsilon)}{(1-2 \delta) \mathrm{e}^{\gamma}} W_{1}<W_{2}$,

but $1-2 \varepsilon>0$ and thus $(1-2 \varepsilon) /(1-2 \delta) \mathrm{e}^{\gamma}$ has positive sign and we can choose $\mathrm{e}^{\gamma}$ large enough so that $(1-$ $2 \varepsilon) /(1-2 \delta) \mathrm{e}^{\gamma}<1$. Thus, we have a line with the slope $(1-2 \varepsilon) /(1-2 \delta) \mathrm{e}^{\gamma}$ and the line $x=y$ defining a cone $\mathrm{OC} 1 \mathrm{in}$ LC which is mapped to UC where $\mathscr{F}^{\gamma}=E_{2}$. A similar calculation shows that $\mathrm{e}^{\gamma}$ can be chosen large enough so that if $W \in \mathrm{OC} 1, E W=E_{2} E_{1} W \in \mathrm{OC} 1$. It follows that starting from every point in $\mathrm{OC} 1$, the action of $\mathscr{F} \gamma$ is defined by alternating multiplications of $E_{1}$ and $E_{2}$, starting with $E_{1}$ and ending with $E_{2}$ if $t$ is even and with $E_{1}$ if $t$ is odd. We have that, if $W \in \mathrm{OC} 1$,

$\left(\mathscr{F}^{\gamma}\right)^{t}(W)= \begin{cases}E_{2} E_{1} \ldots, E_{2} E_{1} W, & t \text { even }, \\ E_{1} E_{2} E_{1} \ldots, E_{2} E_{1} W, & t \text { odd, }\end{cases}$

pairing $E_{1}$ and $E_{2}$, we obtain $E_{1} E_{2}=E$, the operator whose asymptotic properties we studied earlier. We can write

$\left(\mathscr{F}^{\gamma}\right)^{t}(W)= \begin{cases}E^{t} W, & t \text { even, } \\ E_{1} E^{t-1} W, & t \text { odd. }\end{cases}$

By (37), for sufficiently large values of $\gamma, E^{t} W$ approaches a multiple of the dominant eigenvector of $E$; for even values as $t \rightarrow \infty$

$$
\begin{aligned}
\left(F^{\gamma}\right)^{t}(W) & =H\left(\left(\mathscr{F}^{\gamma}\right)^{t}(W)\right) \rightarrow H\left(V_{1}\right) \\
& =\frac{V_{1}}{\sum V_{1}^{i}}:=p^{*} .
\end{aligned}
$$

For odd values of $t$, the same limit is given by $H\left(E_{1} p^{*}\right):=p_{*}$. We have established the unique asymptotic periodicity of $F^{\gamma}$ on OC1 with period 2 and $R=\left\{p^{*}, p_{*}\right\}$.

Furthermore,

$$
\begin{aligned}
F^{\gamma} p^{*} & =\mathscr{A}^{\mathrm{T}} H\left(\left[\begin{array}{ll}
1 & 0 \\
0 & \mathrm{e}^{\gamma}
\end{array}\right] p^{*}\right) \\
& =H\left(\mathscr{A}^{\mathrm{T}}\left[\begin{array}{ll}
1 & 0 \\
0 & \mathrm{e}^{\gamma}
\end{array}\right] p^{*}\right) \\
& =H\left(E_{1} p^{*}\right)=p_{*} .
\end{aligned}
$$

Noting that $E=E_{1} E_{2}$, we can similarly show that $F^{\gamma}\left(p_{*}\right)=p^{*}$.

There remains to carry out the same process in the UC cone and identify the cone OC2 for which $F^{\gamma}$ is asymptotically periodic. To show unique asymptotic periodicity of $F^{\gamma}$ on $\mathrm{OC}=\mathrm{OC} 1 \cup \mathrm{OC} 2$, we must show that the periodic limit for OC2 is the same, up to a permutation, as $R$.

It can be shown either by direct calculation or a symmetry argument that such a cone exists and is defined by the line $x=y$ and the line $\mathrm{e}^{\gamma}(1-2 \varepsilon) /(1-2 \delta)$. This time, $\mathrm{e}^{\gamma}$ appearing in the numerator allows us to choose the slope so that this line lies in UC defining the cone OC2 which is mapped to LC by the action of $F^{\gamma}$. The periodic orbit will be given by a set $R^{\prime}:=$ $\left\{q^{*}, q_{*}\right\}$. We will show that $R^{\prime}$ must be a permutation of $R$. 
Matrices of full rank map cones to cones and one can check that $E_{1}$ and $E_{2}$ have full rank because of the assumption $\varepsilon<1 / 2, \delta<1 / 2$. It is easy to show that $F^{\gamma}$ maps OC2 into OC1. The line with slope $\mathrm{e}^{\gamma}(1-$ $2 \varepsilon) /(1-2 \delta)$ is, by construction, mapped to $x=y$ and it is easy to verify that for sufficiently large values of $\gamma$ any line in UC with a slope less than $\mathrm{e}^{\gamma}(1-$ $2 \varepsilon) /(1-2 \delta)$ is mapped to a line with a slope greater than $\varepsilon \mathrm{e}^{\gamma}+(1-\delta) /(1-\varepsilon) \mathrm{e}^{\gamma}+\delta$ which is easily seen to lie inside OC1. Thus, the image of points in OC2 is a subset of OC1. We have already studied the action of $F^{\gamma}$ for initial points belonging to $\mathrm{OC} 1$ and saw that they are carried asymptotically to $R=\left\{p^{*}, p_{*}\right\}$ (for even values of $t$ to $p^{*}$ and for odd values to $p_{*}$ ).

Consider the action of $\left(F^{\gamma}\right)^{t}$ starting from OC2; after one step, we end up in $\mathrm{OC} 1$ and the $t-1$ remaining steps can be considered as initiated from OC1. If $t$ is even, $t-1$ is odd and vice versa. Thus, the periodic orbit $R^{\prime}$ must be given by $\left\{p_{*}, p^{*}\right\}$, a permutation of $R$ as desired.

To extend the unique asymptotic periodicity of $F^{\gamma}$ to the entire first quadrant, it is sufficient to show that points not belonging to $\mathrm{OC}=\mathrm{OC} 1 \cup \mathrm{OC} 2$ will be mapped into OC, under the action of $F^{\gamma}$. As mentioned before, matrices of full rank preserve cones. In $R^{2}$, a matrix $M$ maps a line with the slope $s$ to a line with the slope

$\frac{m_{21}+m_{22} s}{m_{11}+m_{12} s}$.

This defines a function $s \rightarrow f(s)$ that can be shown to be an increasing function if

$m_{22} m_{11}>m_{12} m_{21}$.

This condition when considered for the linear map $E_{1}$ translates to

$(1-\varepsilon)(1-\delta)>\varepsilon \delta$

which holds since $\varepsilon<1 / 2$ and $\delta<1 / 2$.

$y=0$ is the lower boundary of the cone $\mathrm{NC}$ and if $y=0$ is mapped to $\mathrm{OC} 1$, then any line with a slope greater than zero is mapped to a line with a slope greater than the image of $y=0$ under $E_{1}$ by the above result. For this to hold, we must have

$\frac{\varepsilon}{1-\varepsilon}>\frac{(1-2 \varepsilon)}{(1-2 \delta) \mathrm{e}^{\gamma}}$.
Since $\varepsilon>0$ ( $\mathscr{A}$ is primitive), we can choose $\gamma$ large enough so that the above inequality holds. We can similarly show that the non-oscillating cone in UC is mapped to OC2 for sufficiently large values of $\gamma$. This completes the proof of the theorem.

\section{References}

[1] J.S. Baras, M.R. James, Robust and risk-sensitive output feedback control for finite state machines and hidden Markov models, Technical Research Reports T.R., pp. 94-63, Institute for Systems Research, University of Maryland at College Park.

[2] R.K. Boel, M.R. James, I.R. Petersen, Robustness and risksensitive filtering, Proceedings of the 36th IEEE Conference on Decision and Control, Cat. No. 97CH36124, 1997.

[3] S. Dey, J. Moore, Risk sensitive filtering and smoothing for hidden Markov models, Systems Control Lett. 25 (1995) 361-366.

[4] S. Dey, J. Moore, Risk sensitive filtering and smoothing via reference probability methods, IEEE Trans. Automat. Control 42 (11) (1997) 1587-1591.

[5] M.R. James, J.S. Baras, R.J. Elliot, Risk sensitive control and dynamic games for partially observed discrete-time nonlinear systems, IEEE Trans. Automat. Control 39 (4) (1994) 780-792.

[6] J. Komornik, Asymptotic periodicity of the iterates of weakly constrictive Markov operators, Tohoku Math. J. 38 (1986) 15-17.

[7] J. Losson, M. Mackey, Thermodynamic properties of coupled map lattices, in: S.J. van Strien, S.M. Verduyn Lunel (Eds.), Stochastic and Spatial Structures of Dynamical Systems, North-Holland, Amsterdam, 1996.

[8] H.V. Poor, An Introduction to Signal Detection and Estimation, Springer, Berlin, 1994.

[9] V.R. Ramezani, Ph.D. Dissertation, 2001, Department of Electrical and Computer Engineering, University of Maryland, College Park, Institute for Systems Research Technical Reports PhD 2001-7. http://www.isr.umd.edu/TechReports/ ISR/2001/PhD_2001-7.

[10] V.R. Ramezani, S.I. Marcus, Estimation of hidden Markov models: risk-sensitive filter banks and qualitative analysis of their sample paths, IEEE Trans. Automat. Control 47 (12) (2002) 1999-2009.

[11] A.N. Shiryayev, Probability, Springer, New York, 1984.

[12] J.L. Speyer, C. Fan, R.N. Banava, Optimal stochastic estimation with exponential cost criteria, Proceedings of 31st Conference on Decision and Control, 1992, pp. 2293-2298.

[13] P. Whittle, Risk-Sensitive Optimal Control, Wiley, New York, 1990. 